\title{
The CMS-TOTEM Precision Proton Spectrometer and first physics results
}

\author{
Jonathan Hollar, for the CMS and TOTEM Collaborations* \\ LIP Lisbon \\ E-mail: jjhollarecern.ch
}

\begin{abstract}
Proton tagged photon-photon and gluon-gluon interactions represent a unique way to study the Standard Model, and to search for new physics beyond the Standard Model, in proton-proton collisions at the LHC. The CT-PPS project began operating near-beam detectors housed in Roman Pots during 2016, to detect the forward scattered protons in these processes. The 2016 data was used to develop techniques for alignment and beam optics corrections, and applied to an analysis of $\gamma \gamma \rightarrow \ell^{+} \ell^{-}$production. This resulted in the first observation of this process with tagged protons in the mass range $m\left(\ell^{+} \ell^{-}\right)>110 \mathrm{GeV}$. Several upgrades of the detectors since 2016 provide enhanced sensitivity for new physics searches and Standard Model measurements.
\end{abstract}

The 39th International Conference on High Energy Physics (ICHEP2018)

4-11 July, 2018

Seoul, Korea

${ }^{*}$ Speaker. 


\section{Introduction}

The CMS-TOTEM Precision Proton Spectrometer (CT-PPS) [1] began data-taking operations during 2016. In the initial data-taking phase, the existing silicon strip tracking detectors of the TOTEM collaboration were used [2,3]. These detectors are housed in movable Roman Pot (RP) devices, allowing the detectors to approach within a few $\mathrm{mm}$ of the beam in the horizontal direction. The data acquisition and reconstruction software were fully integrated with the DAQ and software of the central CMS [4] detectors. During 2016, a luminosity of approximately $15 \mathrm{fb}^{-1}$ was collected with the detectors operational and the RPs inserted. The results discussed here correspond to 9.4 $\mathrm{fb}^{-1}$, recorded with consistent beam optics conditions before September 2016.

\section{Alignment and optics corrections}

In order to align the detectors, a two step procedure is used [5]. First, special calibration fills are taken, with both horizontal and vertical RPs inserted more closely than normal. This allows the vertical RPs to collect a sample of elastic scattering events ( $p p \rightarrow p p$ ), to perform an absolute alignment. The horizontal RPs are aligned with respect to the vertical RPs using tracks passing through the region where they overlap. In a second step, fill-by-fill alignment corrections are derived for normal physics runs, by assuming the distribution of physics protons is the same in all fills, and aligning the distributions of the track x positions.

After deriving the alignment corrections, the measurement of the proton fractional momentum loss, $\xi$, requires a precise knowledge of the LHC beamline optics, particularly the horizontal dispersion $D_{x}$. The optical functions can be expressed as elements of the single-pass transport matrix $T(s, \xi)$. Distributions of proton tracks detected in the Roman Pots are used to constrain the elements of this matrix. A method based on studying the vertical distribution of tracks vs. the horizontal position is used [6]. By finding the point of the most narrow "pinch" in the vertical distribution, corresponding to the point at which the vertical effective length optical function $\left(L_{y}(\xi)\right)$ vanishes, the dispersion $D_{x}$ can be estimated.

\subsection{Analysis of $\gamma \gamma \rightarrow \ell^{+} \ell^{-}$production}

Having determined the alignment and optics corrections, the protons detected in the CT-PPS detectors can be combined with objects reconstructed in the central CMS detectors. One of the cleanest processes with intact forward protons at the LHC is dilepton production $\left(\mu^{+} \mu^{-}\right.$or $\left.e^{+} e^{-}\right)$ via $\gamma \gamma$ fusion. This signature is therefore studied for an initial analysis using the CT-PPS data [7]. In order to increase the statistics available for the analysis, both fully exclusive events, in which both protons remain intact, and single dissociation events, in which one of the two protons remains intact, are considered as signal. For each event, the value of the fractional momentum loss of the scattered proton is estimated from the leptons as:

$$
\xi\left(\ell^{+} \ell^{-}\right)=\frac{1}{\sqrt{s}}\left[p_{T}\left(\ell^{+}\right) e^{ \pm \eta\left(\ell^{+}\right)}+p_{T}\left(\ell^{-}\right) e^{ \pm \eta\left(\ell^{-}\right)}\right],
$$

where the two solutions for $\pm \eta$ correspond to the protons moving in the $\pm z$ direction.

In the central detectors, a sample of dileptons enhanced in $\gamma \gamma$ production is first selected. This is accomplished by requiring that the two leptons be back-to-back in the azimuthal angle $\phi$, 
and that no additional charged tracks are reconstructed near the dilepton vertex. The remaining backgrounds are expected to arise mainly from real dilepton events, produced via the Drell-Yan or double dissociation mechanisms. The probability for these backgrounds to coincide with a matching proton in CT-PPS, is estimated from samples of $Z \rightarrow l^{+} l^{-}$events in data.

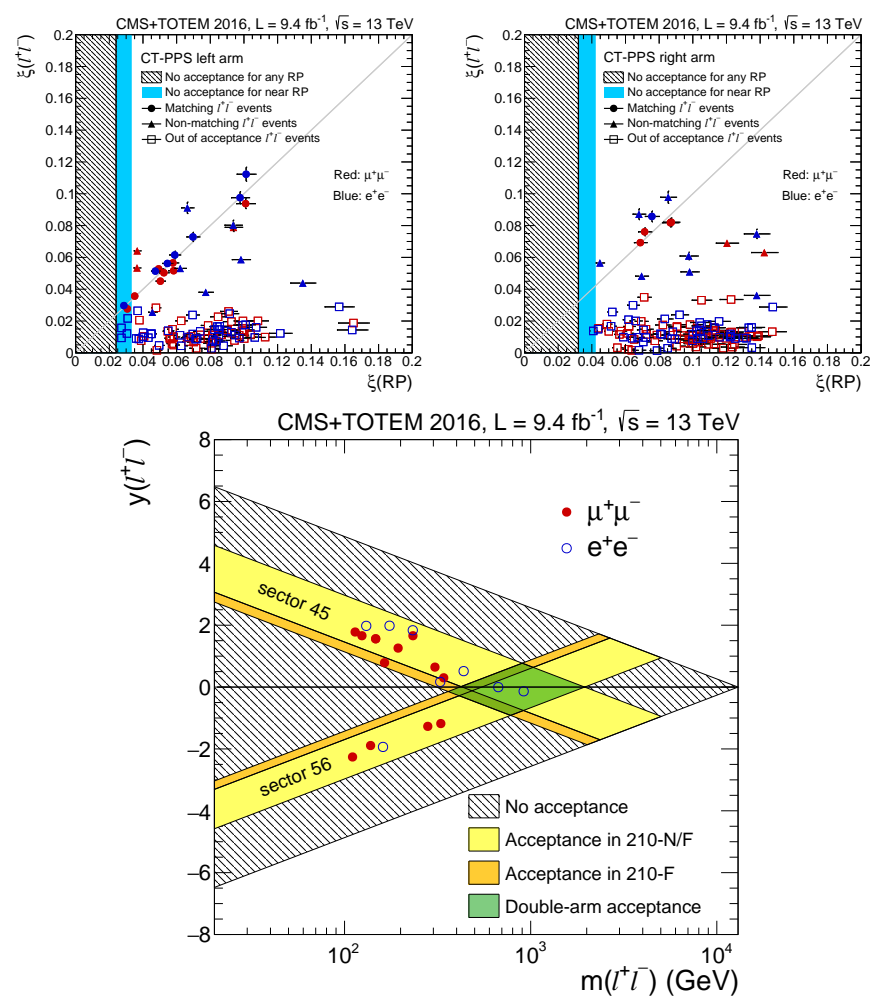

Figure 1: Above: The distribution of $\xi\left(\ell^{+} \ell^{-}\right)$vs. $\xi(\mathrm{RP})$ for the sample of dilepton events passing the final analysis selection. The distributions are shown separately for the Roman Pots in the LHC sectors 45 (left) and 56 (right). Below: The distribution of events with matching $\xi$ values in the plane of the dilepton mass and rapidity. The yellow bands indicate the acceptance for detecting 1 proton, the green diamond indicates the region where both protons can be detected if they remain intact (figures from Ref. [7]).

In Figure 1, the correlation between $\xi(\mathrm{RP})$ and $\xi\left(\ell^{+} \ell^{-}\right)$is shown for each of the two arms, for the events passing the final selection. The events with low $\xi\left(\ell^{+} \ell^{-}\right)$are incompatible with producing protons within the acceptance of the RPs, and are expected to be dominated by random coincidences with pileup protons or beam backgrounds. In the region compatible with the RP acceptance, a total of 20 events (12 in the dimuon channel and 8 in the dielectron channel) are found that pass the matching criteria. The expected backgrounds are $1.49 \pm 0.07$ (stat.) \pm 0.53 (syst.) events (in the $\mu^{+} \mu^{-}$channel) and 2.36 \pm 0.09 (stat.) \pm 0.47 (syst.) events (in the $e^{+} e^{-}$channel). The significance over the background-only hypothesis is estimated to be $5.1 \sigma$, including systematic uncertainties. This represents the first observation of single proton-tagged $\gamma \gamma \rightarrow \ell^{+} \ell^{-}$production in this mass range.

Figure 1 shows the distribution of the matching events in the dilepton mass-rapidity plane. The selected events are consistent with the acceptance for single-tagged production. No events have both protons detected; this is consistent with SM prediction in a region where the strip detectors 
had high efficiency throughout the year $(\xi>0.05)$. Two events have kinematics which would have allowed both protons to be detected if they remained intact. These were examined and no activity or signs of reconstruction failures were found in the arm with no proton track; this is consistent with the two events being either single dissociation or backgrounds.

\section{Prospects}

The present results demonstrate that the CT-PPS detectors work as expected, and that the procedures for deriving the corrections to perform physics analyses are in place. Since 2016, the detectors have been further upgraded to enhance the physics performance. The silicon strip detectors have been replaced with 3D pixel tracking detectors, which, in addition to providing improved radiation hardness, allow the reconstruction of multiple tracks within a single bunch crossing. In addition, diamond precision timing detectors have been installed, which will allow reconstruction of the collision vertex position from the time of flight difference of the two protons.

\section{Conclusions}

Starting in the 2016 LHC run, the CT-PPS project began operating near-beam detectors housed in Roman Pots. This demonstrated for the first time the feasibility of using such detectors continuously in a high-luminosity hadron collider environment. The 2016 data was used to develop procedures for alignment and optics corrections, and apply them to a first physics measurement of $\gamma \gamma \rightarrow l^{+} l^{-}$production with at least one intact proton detected. This resulted in the first observation of this process with proton tags at high masses. Since 2016, detector upgrades have been performed to enhance both the tracking and timing capabilities of CT-PPS. This will allow a broad physics program of Standard Model and Beyond Standard Model studies with forward protons in run 2 of the LHC and beyond.

\section{References}

[1] M. Albrow et al. [CMS and TOTEM Collaborations], CERN-LHCC-2014-021, TOTEM-TDR-003, CMS-TDR-13.

[2] G. Ruggiero et al., Nucl. Instrum. Meth. A 604, 242 (2009). doi:10.1016/j.nima.2009.01.056

[3] G. Antchev et al. [TOTEM Collaboration], Int. J. Mod. Phys. A 28, 1330046 (2013) doi:10.1142/S0217751X13300469 [arXiv:1310.2908 [physics.ins-det]].

[4] S. Chatrchyan et al. [CMS Collaboration], JINST 3, S08004 (2008). doi:10.1088/1748-0221/3/08/S08004

[5] J. Kaspar, CERN-TOTEM-NOTE-2017-001.

[6] F. Nemes, CERN-TOTEM-NOTE-2017-002.

[7] A. M. Sirunyan et al. [CMS and TOTEM Collaborations], JHEP 1807, 153 (2018) doi:10.1007/JHEP07(2018)153 [arXiv:1803.04496 [hep-ex]]. 